\title{
Laparoscopic transabdominal pre-peritoneal obturator hernia repair: An operative technique
}

\author{
Mihir M Shah, Jean-Pierre Marctucci, Kevin El-Hayek, \\ Ashley Valentine, David Baringer
}

\begin{abstract}
Introduction: Obturator hernia is a rare form of hernia that tends to occur in elderly emaciated women, and is typically associated with chronic diseases. Delay in clinical presentation and difficulty in diagnosis contribute significantly to the morbidity and mortality of this disease which can approach $13-40 \%$. Treatment is always surgical and in most cases it is performed via an emergent laparotomy. Case Report: We report a case of a 59-year-old female who presented with right labial swelling and was diagnosed with an obturator hernia based on history and physical exam. An elective laparoscopic transabdominal pre-peritoneal (TAPP) approach was used to repair a nonstrangulated obturator hernia. We highlight the role of a laparoscopic repair and describe the technique used. Conclusion: A high index of suspicion for female patients with vaginal swelling or pain may prevent the eventual morbidity and mortality associated with stage 3 obturator hernia with incarceration. In this case report, we present a case of early detection of stage 1 obturator hernia which was successfully repaired using concepts of a TAPP approach. Outpatient laparoscopic repair of obturator
\end{abstract}

Mihir M Shah ${ }^{1}$, Jean-Pierre Marctucci ${ }^{1}$, Kevin El-Hayek², Ashley Valentine ${ }^{3}$, David Baringer ${ }^{2}$

Affiliations: ${ }^{1} \mathrm{MD}$, Resident, General Surgery, Cleveland Clinic Foundation, Cleveland, Ohio, USA; ${ }^{2} \mathrm{MD}$, Fellow, General Surgery, Cleveland Clinic Foundation, Cleveland, Ohio, USA; 'PA-C, Physician's Assistant, General Surgery, Cleveland Clinic Foundation, Cleveland, Ohio, USA.

Corresponding Author: Mihir M Shah, 35 Severance Circle, Apt 311, Cleveland Heights, Ohio, USA, 44118; Phone: (+1) 215-200-6256; Fax: (+1) 216-445-7653 E-mail: mihir253@yahoo.co.in

Received: 30 July 2012

Accepted: 16 November 2012

Published: 01 June 2013 hernia resulted in symptomatic relief at a threeweek follow-up. Laparoscopic approach may be useful for the repair of a non-strangulated obturator hernia, particularly if diagnosed in its early stages.

Keywords: Obturator hernia, Obturator foramen, Obturator nerve, Laparoscopic transsabdominal pre-peritoneal repair

$$
* * * * * * * * *
$$

Shah MM, Marctucci JP, El-Hayek K, Valentine A, Baringer D. Laparoscopic transabdominal preperitoneal obturator hernia repair: Operative technique and review of literature. International Journal of Case Reports and Images 2013;4(6):312-315.

$$
* * * * * * * * *
$$

doi:10.5348/ijcri-2013-06-320-CR-5

\section{INTRODUCTION}

Obturator hernia is usually diagnosed among septuagenarian women with the typical clinical presentation of an acute bowel obstruction [1-3]. An Obturator hernia traverses a defect in the anterosuperior aspect of the obturator membrane, which covers the obturator foramen and then passes via the obturator canal containing obturator vessels and nerves [4]. A body mass index (BMI) below 18.5 and multiparity, which increases the obturator foramen diameter, are among the most important risk factors for obturator hernia [5]. In the past, establishing a correct preoperative diagnosis was difficult. However, this has become easier with advancements in imaging technology $[2,6,7]$. Emergency laparotomy remains a common therapeutic approach, as most cases present with incarcerated or strangulated bowel in an acutely ill patient [8]. We report a case of a 59-year-old female 
who was diagnosed clinically with an obturator hernia. Therefore, she underwent a successful elective laparoscopic repair for a reducible right obturator hernia.

\section{CASE REPORT}

Our patient is a 59-year-old female who presented with right vaginal "swelling" and pain. She was seen by multiple physicians prior to our evaluation, whereby she was diagnosed with chronic labial edema. On examination, her BMI was 18.7 and she presented with a reducible mass above her right labia, consistent with an obturator hernia. No preoperative imaging was obtained as her diagnosis was made clinically. She had a past surgical history of appendectomy via an ventral midline incision, but was otherwise healthy. Therefore, she was offered an elective laparoscopic repair via the transabdominal route.

\section{OPERATIVE TECHNIQUE AND RESULTS}

The patient was placed in lithotomy position to allow for access to her genital region to assist with reduction of hernia contents during the laparoscopic repair. A foley catheter was placed for bladder decompression. Port placement was performed as a standard transabdominal pre-peritoneal approach, with a $10 \mathrm{~mm}$ midline port and two $5 \mathrm{~mm}$ lateral working ports placed at the level of the umbilicus and mid-clavicular line. The peritoneum was incised in a medial to lateral fashion from the medial umbilical ligament to the right flank. An important technical aspect is to keep the peritoneal incision as superior to the anterior superior iliac spine (ASIS) as possible to allow for loose re-approximation after mesh placement. Once the peritoneal flap was created, blunt dissection in the pre-peritoneal space to the level of Cooper's ligament was performed. The round ligament was divided between clips. Using direct pressure above the labia in the region corresponding to the patient's preoperative bulge and tenderness, the hernia was identified. While no bowel was protruding through, pre-peritoneal fat was herniating through the obturator defect, medial to the vessels and nerve. As seen in Figure 1, the obturator canal runs inferior to Cooper's ligament, and care must be taken not to injure the iliac vessels or nerves during dissection. Once the hernia contents were fully reduced and the anatomy delineated, a 2x2 cm defect was identified in the obturator canal. A $6 \times 6$ inches lightweight polyester mesh was introduced into the abdomen to fully cover this defect (Figure 2). Because this patient had no direct or indirect component, the superior aspect of the mesh was secured to Cooper's ligament at the midline. The superior aspect of the mesh was then secured laterally with permanent tacks. However, the inferior portion is not secured with tacks due to the proximity to nerves and vessels (Figure 3). Therefore, fibrin sealant was used to secure this portion of the mesh. Adequate coverage was confirmed by external pressure over the obturator region. The peritoneal flap was reapproximated with permanent tacks to avoid intraabdominal exposure of the mesh. The $10 \mathrm{~mm}$ port was closed with a o absorbable suture in a figure of eight fashion.

Operating room time was 60 minutes. The patient was discharged the same day post-operatively and on three week follow-up, she was symptom free.

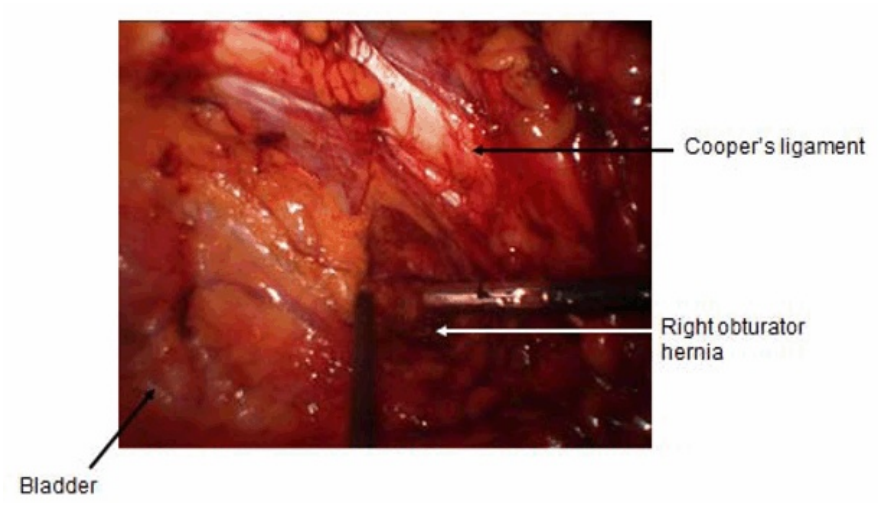

Figure 1: Pre-peritoneal exposure of right obturator hernia.

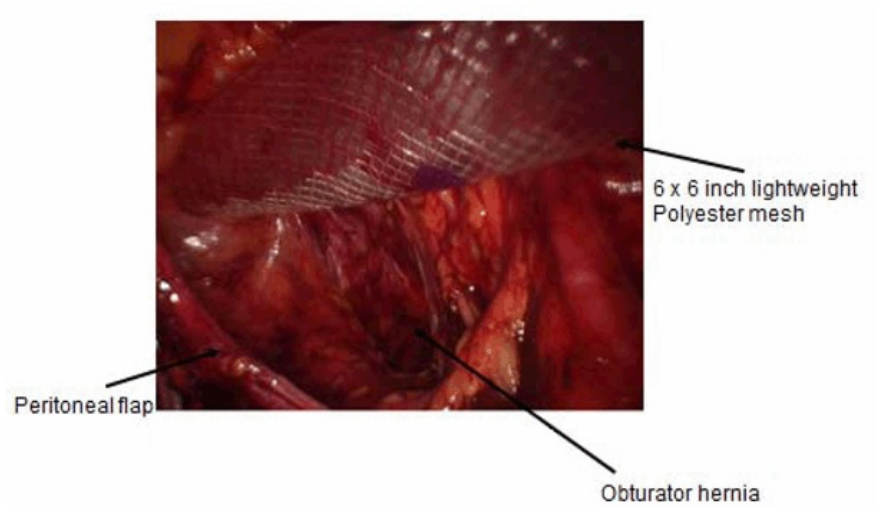

Figure 2: Pre-peritoneal position of the mesh.

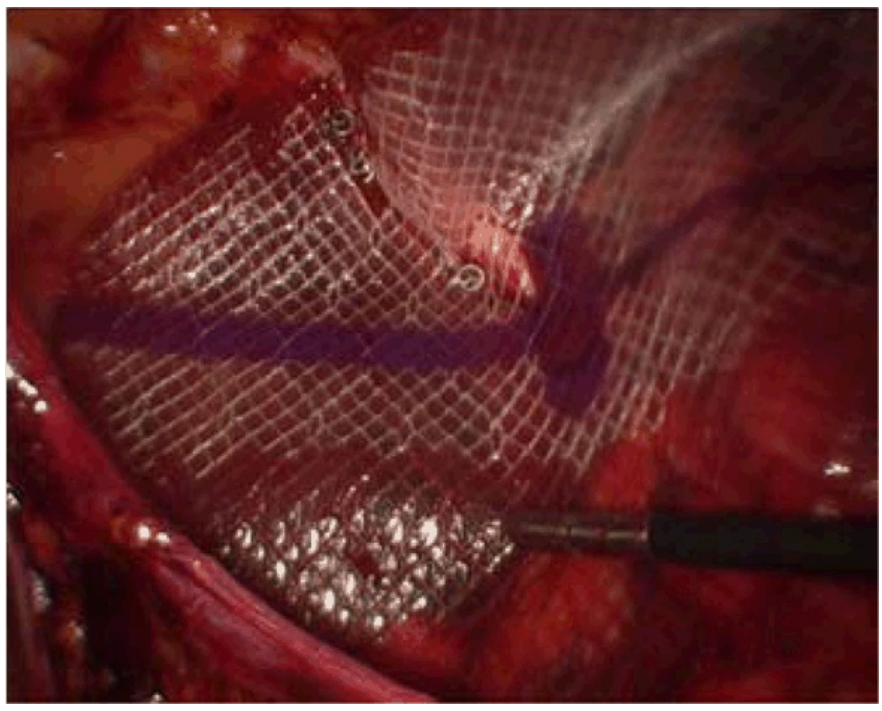

Figure 3: Final position of pre-peritoneal mesh with permanent tacks securing mesh to Cooper's ligament. 


\section{DISCUSSION}

An obturator hernia is a rare pelvic hernia representing less than $1 \%$ of all abdominal wall hernias [9, 10]. Arnaud de Ronsil of France first described obturator hernia in 1724, and Obre is credited with its successful repair in 1851 [9]. Approximately, 676 such hernias were reported in literature by 2001 [11]. Six percent of the cases are bilateral. However, there is a right sided predominance secondary to sigmoid colon protection of the obturator foramen on the left side [3]. Conditions that promote obturator hernia include a congenitally large obturator foramen, increased intraabdominal pressure (COPD, constipation, pregnancy, multiparity, or ascites), and increase in size of the obturator foramen relative to the surrounding adipose tissue resulting from dramatic weight loss [10, 12]. Obturator hernia is nine times more common in females due to their wider pelvis, more triangular obturator canal opening and greater transverse diameter.

The obturator foramen consists of the superior pubic ramus on the superior pubic body, inferior ramus on the medial, and ischial ramus on the inferior side [13]. The obturator foramen in the anteromedial pelvic wall is positioned in the inferior portion of acetabulum, typically located between the origin of the medial umbilical and lateral umbilical fold in the abdominal cavity. The obturator canal (2-3 cm in length, $0.3-0.5 \mathrm{~cm}$ in diameter) within the obturator foramen is protected by the obturator membrane, except in the superior portion where the obturator nerve and vessels penetrate the membrane. The obturator membrane, a consecutive structure of the periosteum, is located between the internal and external obturator muscles and gains its significance in determining the predilection sites and the stages of obturator hernia formation [14]. Three stages of obturator hernia formation are described as follows:

The first stage is called 'pilot tags' in which the preperitoneal connective tissue and fat protrude into the pelvic orifice of the obturator canal without internal dimpling of the peritoneum. The second stage is when the peritoneal dimpling begins in the obturator canal's pelvic orifice to form the peritoneal sac. The third stage is when herniation of the intra-abdominal organ begins and symptoms occur $[1,15]$.

Chronic symptoms arise mostly due to obturator nerve compression [1, 16]. This compression causes altered sensation at the inguinal crest and the anteromedial region of the thigh. Clinically, this pain pattern is called the Howship-Romberg sign, when the pain characteristically exacerbates with extension, adduction or inward rotation of the thigh. Flexion may relieve the pain. The Howship-Romberg sign is present in $15-50 \%$ of the cases and is quite specific for obturator hernia [10]. Since osteoarthritis is common in the elderly, this sign is often overlooked or misinterpreted, leading to neurological or orthopedic referral. The Hannington-Kiff sign is present when the adductor reflex is absent, but the patella reflex is present. The adductor reflex is elicited by placing the index finger across the adductor muscle about 2 inches above the knee and percussing onto the extended finger with a patella hammer. The muscle contraction is seen or felt. The Hannington-Kiff sign is reportedly more specific than the Howship-Romberg sign [1].

Conventional radiographs may show gas bubbles at the obturator foramen. However, this is only present in the third stage of obturator hernia when the hernia sac contains bowel or when an abscess is present due to perforation. Ultrasound is frequently inaccurate due to the deep location of the obturator canal, but it can be very useful and reliable in cases of strangulation [17, 18]. Computed tomography (CT) scan is the preferred imaging modality for evaluation of obturator hernia and its complications $[1,6,16,17]$. Recent studies suggest a better outcome with early diagnosis of incarcerated hernias with a CT scan [6]. In our case, the diagnosis of obturator hernia was made based on history and physical examination.

\section{CONCLUSION}

Obturator hernia is a rare diagnosis that predominates in elderly, thin females. When detected early based on clinical signs and symptoms, patients may undergo elective, laparoscopic repair. A high index of suspicion for female patients with vaginal swelling or pain may prevent the eventual morbidity and mortality associated with stage 3 obturator hernia with incarceration. Herein, we present a case of early detection of stage 1 obturator hernia which was successfully repaired using concepts of a transabdominal pre-peritoneal approach.

$$
* * * * * * * * *
$$

\section{Author Contributions}

Mihir Shah - Conception and design, Acquisition of data, Analysis and interpretation of data, Drafting the article, Critical revision of the article, Final approval of the version to be published

Jean-Pierre Martucci - Conception and design, Acquisition of data, Analysis and interpretation of data, Drafting the article, Final approval of the version to be published

Kevin El-Hayek - Conception and design, Acquisition of data, Analysis and interpretation of data, Drafting the article, Critical revision of the article, Final approval of the version to be published

Ashley Valentine - Acquisition of data, Drafting the article, Final approval of the version to be published David Baringer - Conception and design, Acquisition of data, Analysis and interpretation of data, Drafting the article, Critical revision of the article, Final approval of the version to be published

\section{Guarantor}

The corresponding author is the guarantor of submission. 


\section{Conflict of Interest}

Authors declare no conflict of interest.

\section{Copyright}

(C) Mihir Shah et al. 2013; This article is distributed under the terms of Creative Commons Attribution 3.0 License which permits unrestricted use, distribution and reproduction in any means provided the original authors and original publisher are properly credited. (Please see www.ijcasereportsandimages.com/copyright-policy.php for more information.)

\section{REFERENCES}

1. Losanoff JE, Richman BW, Jones JW. Obturator hernia. J Am Coll Surg 2002;194(5):657-63.

2. Bergstein JM, Condon RE. Obturator hernia: current diagnosis and treatment. Surgery 1996;119(2):133-6.

3. Lo CY, Lorentz TG, Lau PW. Obturator hernia presenting as small bowel obstruction. Am J Surg 1994;167(4):396-8.

4. O'Connell G, Cole A. Obturator hernia: diagnosis through medical imaging. Australas Radiol 1995;39(3):306-8.

5. Rodríguez-Hermosa JI, Codina-Cazador A, MarotoGenover A, et al. Obturator hernia: clinical analysis of 16 cases and algorithm for its diagnosis and treatment. Hernia 2008;12(3):289-7.

6. Kammori M, Mafune K, Hirashima T, et al. Fortythree cases of obturator hernia. Am J Surg 2004;187(4):549-52.

7. Ijiri R, Kanamaru H, Yokoyama H, Shirakawa M, Hashimoto H, Yoshino G. Obturator hernia: the usefulness of computed tomography in diagnosis. Surgery 1996;119(2):137-40.
8. Ziegler DW, Rhoads JE Jr. Obturator hernia needs a laparotomy, not a diagnosis. Am $J$ Surg 1995;170(1):67-8.

9. Bjork KJ, Mucha P Jr, Cahill DR. Obturator hernia. Surg Gynecol Obstet 1988;167(3):217-2.

10. Yip AW, AhChong AK, Lam KH. Obturator hernia: a continuing diagnostic challenge. Surgery 1993;113(3):266-9.

11. Martinez Insua C, Costa Pereira JM, Cardoso de Oliveira M. Obturator hernia: the plug technique. Hernia 2001;5(3):161-3.

12. Schmidt PH, Bull WJ, Jeffery KM, Martindale RG. Typical versus atypical presentation of obturator hernia. Am Surg 2001;67(2):191-5.

13. Skandalakis LJ, Androulakis J, Colborn GL, Skandalakis JE. Obturator hernia. Embryology, anatomy, and surgical applications. Surg Clin North Am 2000;80(1):71-84.

14. Kim JJ, Jung $\mathrm{H}$, Oh SJ, et al. Laparoscopic Transabdominal Preperitoneal Hernioplasty of Bilateral Obturator Hernia. Surg Laparosc Endosc Percutan Tech 2005;15(2):106-9.

15. Yokoyama Y, Yamaguchi A, Isogai M, Hori A, Kaneoka Y. Thirty-six cases of obturator hernia: does computed tomography contribute to postoperative outcome? World J Surgery 1999;23(2):214-6.

16. Mantoo SK, Mak K, Tan TJ. Obturator hernia: diagnosis and treatment in the modern era. Singap Med J 2009;50(9):866-70.

17. Terada R, Ito S, Kidogawa H, Kashima K, Ooe H. Obturator hernia: the usefulness of emergent computed tomography for early diagnosis. J Emerg Med 1999;17(5):883-6.

18. Yokoyama T, Munakata Y, Ogiwara M, Kamijima T, Kitamura H, Kawasaki S. Preoperative diagnosis of strangulated obturator hernia using ultrasonography. Am J Surg 1997;174(1):76-8.
Access full text article on other devices

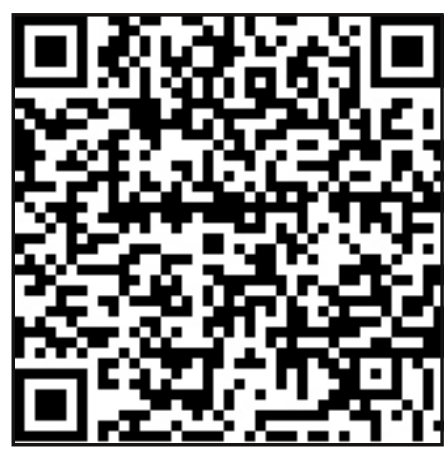

Access PDF of article on other devices

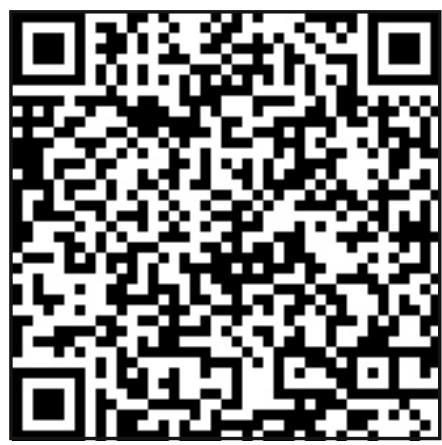

